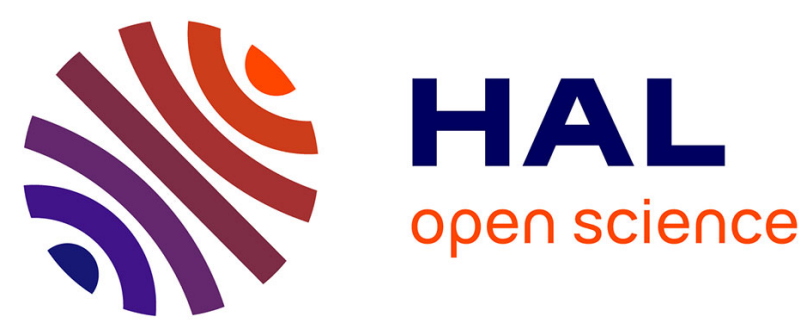

\title{
Source Scanning Technique for Simulating TBL-Induced Vibrations Measurements
}

\author{
Mathieu Aucejo, Laurent Maxit, Jean-Louis Guyader
}

\section{To cite this version:}

Mathieu Aucejo, Laurent Maxit, Jean-Louis Guyader. Source Scanning Technique for Simulating TBL-Induced Vibrations Measurements. Flinovia - Flow Induced Noise and Vibration Issues and Aspects, 2014. hal-02121068

\section{HAL Id: hal-02121068 https://hal.science/hal-02121068}

Submitted on 6 May 2019

HAL is a multi-disciplinary open access archive for the deposit and dissemination of scientific research documents, whether they are published or not. The documents may come from teaching and research institutions in France or abroad, or from public or private research centers.
L'archive ouverte pluridisciplinaire HAL, est destinée au dépôt et à la diffusion de documents scientifiques de niveau recherche, publiés ou non, émanant des établissements d'enseignement et de recherche français ou étrangers, des laboratoires publics ou privés. 


\title{
Source Scanning Technique for simulating TBL-induced vibrations measurements
}

\author{
Mathieu Aucejo, Laurent Maxit and Jean-Louis Guyader
}

\begin{abstract}
Measuring the vibro-acoustic response of structures subjected to a turbulent boundary layer (TBL) excitation in operating conditions remains an open issue for experimenters. Generally, in situ measurements, e.g. flight tests, underwater measurements and wind tunnel measurements are carried out, although they require expensive facilities. As an alternative to in situ measurements, experimental simulations in a laboratory environment have been developed. The main issue of these substitute experiments is the synthesis of an excitation field equivalent to TBL wallpressure fluctuations with the help of standard transducers (shakers, loudspeakers, etc.). In the present paper, we propose an alternate off-line methodology to deal with the experimental simulation of vibrations induced by a spatially correlated random pressure field, such as TBL excitation. The proposed methodology is called Source Scanning Technique (SST) and relies on two main features: a wall-pressure plane wave expansion of the target random wall-pressure field and two identification steps based on the concept of synthetic array to simulate TBL-induced vibrations from a set of transfer functions. In the present paper, the theoretical description of the SST and its experimental implementation are detailed.
\end{abstract}

Mathieu Aucejo

Structural Mechanics and Coupled Systems Laboratory, Cnam Paris, 2 rue Conté 75003 Paris, e-mail: mathieu.aucejo@cnam.fr

Laurent Maxit

Vibration and Acoustics Laboratory, INSA de Lyon, Bâtiment St. Exupéry 25 bis av. Jean Capelle, 69621 Villeurbanne cedex - France e-mail: laurent.maxit@insa-lyon.fr

Jean-Louis Guyader

Vibration and Acoustics Laboratory, INSA de Lyon, Bâtiment St. Exupéry 25 bis av. Jean Capelle, 69621 Villeurbanne cedex - France e-mail: jean-louis.guyader@insa-lyon.fr 


\section{Introduction}

Measuring Turbulent Boundary Layer (TBL) induced vibrations is of particular interest in numerous transportation applications. From a practical point of view, in situ measurements, e.g. flight tests [1], underwater measurements [2] and wind tunnel measurements [3] are generally carried out. However, these experimental set-ups are expensive due to the equipment and resources needed. Furthermore, measurements are difficult to perform because of background noise levels. It is thus of considerable interest to develop substitute experiments to assess TBL-induced vibrations under laboratory conditions. Nevertheless, very few studies have been carried out in the last few decades. One of the first studies on this topic was proposed by Fahy in 1966 [4], in which several ways of simulating TBL wall-pressure fluctuations such as arrays of shakers or loudspeakers have been mentioned. Unfortunately, these approaches had not been assessed experimentally because of the practical difficulties of implementing these solutions at that time.

The practical feasibility of using an array of suitably driven shakers has been studied by Robert [5], while the real-time synthesis of spatially correlated random pressure fields with a near-field array of appropriately-driven loudspeakers was studied by Elliot et al. [6] and Maury and Bravo [7, 8]. Apart from these works, other semi-experimental techniques have been proposed. For instance, it is worth citing the work of Audet et al. [9] based on the standard formulation of random vibration theory and the measurement of the required transfer functions and the work proposed by Robin et al. [10] based on the planar NAH.

In the present paper, we propose an alternate off-line methodology called Source Scanning Technique (SST) to investigate structural vibrations induced by random pressure fields such as TBL excitation $[13,14]$. The aim is to overcome the difficulty of generating TBL excitation experimentally and gain the practical advantage of performing a substitute experiment based on acoustic excitation. For this purpose, the methodology developed in this paper relies on two main features: a wall-pressure plane wave expansion of the target random wall-pressure field and two identification steps based on the concept of synthetic array to simulate TBL-induced vibrations from a set of transfer functions. Practically, SST requires the generation of a set of uncorrelated wall-pressure fields corresponding to those of propagating and evanescent acoustic plane waves. For this purpose, a single moving monopole-like source simulating a full array of acoustic monopoles is used.

In the next of the paper, the theoretical description of SST and its experimental implementation are presented. A particular attention is paid to the definition of the grid covered by the monopole source (number of nodes, dimension and distance from the observation area) as well as the design of the test bench used to assess the validity of SST. This test bench being developed for validation purposes only, it can not be used as it is in an industrial context. That is why, a test bench is proposed for industrial applications in the last part of the paper. 


\section{Basic principles of SST}

In room acoustics, it is well-known that a diffuse sound field can be thought as an uncorrelated isotropic superposition of acoustic plane waves [11]. As the diffuse sound field, the TBL excitation is a partially space-correlated random pressure field. Consequently, it can also be modelled as a superposition of uncorrelated wall-pressure plane waves.

\subsection{Modelling of TBL excitation as a superposition of uncorrelated wall plane waves}

As a random process, the TBL excitation classically is modelled from the crossspectral density (CSD) function of the wall-pressure fluctuations. Here, the basic idea is to represent the TBL CSD function as a superposition of uncorrelated wallpressure plane waves.

By definition, the pressure of a wall-pressure plane wave $P_{r s}$ of wavenumbers $\left(k_{r}, k_{s}\right)$ is:

$$
P_{r s}(x, y, t)=A_{r s}(t) \mathrm{e}^{j k_{r} x+j k_{s} y}
$$

where $A_{r s}(t)$ is a random variable corresponding to the amplitude of a wall-pressure plane wave of wavenumbers $\left(k_{r}, k_{s}\right)$.

The corresponding CSD function $S_{P_{r S} P_{r s}}$ between 2 points is therefore:

$$
S_{P_{r s} P_{r s}}\left(\xi_{x}, \xi_{y}, \omega\right)=S_{A_{r s} A_{r s}}(\omega) \mathrm{e}^{j k_{r} \xi_{x}+j k_{s} \xi_{y}}
$$

where $\xi_{x}$ and $\xi_{y}$ are the spatial shifts between 2 points along x-axis and y-axis respectively, $S_{A_{r s} A_{r s}}(\omega)$ is the auto-spectral density (ASD) function of the wallpressure plane wave amplitude and $\omega$ is the angular frequency.

Let us suppose now a rigid surface impacted by a set of uncorrelated wallpressure plane waves. The total pressure $p(x, y, t)$ at point $(x, y)$ of the rigid surface is thus given by:

$$
p(x, y, t)=\sum_{r, s} P_{r s}(x, y, t)
$$

As the wall-pressure plane waves are supposed uncorrelated, $S_{A_{r s} A_{r^{\prime} s^{\prime}}}(\omega)=0$ if $r \neq r^{\prime}$ and $s \neq s^{\prime}$. Consequently, the CSD function of the total pressure is:

$$
S_{p p}\left(\xi_{x}, \xi_{y}, \omega\right)=\sum_{r, s} S_{A_{r s} A_{r s}}(\omega) \mathrm{e}^{j k_{r} \xi_{x}+j k_{s} \xi_{y}}
$$

To obtain a pressure field compatible with the TBL excitation, the CSD function of the total pressure has to be scaled on the TBL CSD function. 
In [14], it has been shown that TBL excitation can be represented as a superposition of uncorrelated wall-pressure plane waves, if the ASD function $S_{A_{r s} A_{r s}}(\omega)$ of each wall-pressure plane wave satisfies:

$$
S_{A_{r s} A_{r s}}(\omega)=\frac{\Gamma_{p p}\left(k_{r}, k_{s}, \omega\right) \Delta k_{r} \Delta k_{s}}{4 \pi^{2}}
$$

\subsection{Vibration response to a TBL excitation}

Let us consider a structure subjected to a homogeneous stationary TBL excitation. It has been shown in [14] that the ASD function of the velocity $S_{v v}(Q, \omega)$ at point $\mathrm{Q}$ of the structure can be obtained from the uncorrelated wall-pressure plane waves expansion described previously. After some calculations based on the classical random vibration theory [12] and the wall-pressure plane waves expansion, it readily comes:

$$
S_{v v}(Q, \omega)=\sum_{r, s} S_{A_{r s} A_{r s}}(\omega)\left|H_{v}\left(Q, k_{r}, k_{s}, \omega\right)\right|^{2}
$$

where the transfer function $H_{v}\left(Q, k_{x}, k_{y}, \omega\right)$ is the structural response at point Q excited by a wall-pressure plane wave of unit amplitude.

Consequently, the experimental process has to be divided in two main steps to compute the response $S_{v v}(Q, \omega)$, namely the measurement of the transfer function $H_{v}\left(Q, k_{r}, k_{s}, \omega\right)$ and their post-processing according to Eq. (6). However, since $H_{v}\left(Q, k_{r}, k_{s}, \omega\right)$ is the response of the structure at point $Q$ to a wall-pressure plane wave of unit amplitude, the main question that arises here is: How to generate a wall-pressure plane wave of unit amplitude?

\subsection{Generation of wall-pressure plane waves}

A natural approach to obtain a wall-pressure field of plane wave type is to generate an acoustic plane wave. However, as illustrated in Fig. 1, it is necessary for a TBL excitation to generate wall-pressure fields corresponding to those of propagating and evanescent acoustic plane waves. Actually, the nature of the wall-pressure field to reproduce only depends on the values of the wavenumbers $k_{r}$ and $k_{s}$ with respect to the acoustic wavenumber $k_{0}$.

Unfortunately, evanescent acoustic plane waves are difficult to generate in practice. That is why, an array of acoustic monopoles is used instead to simulate the required near-field interferential conditions. A schematic representation of such a device is proposed in Fig. 2. 


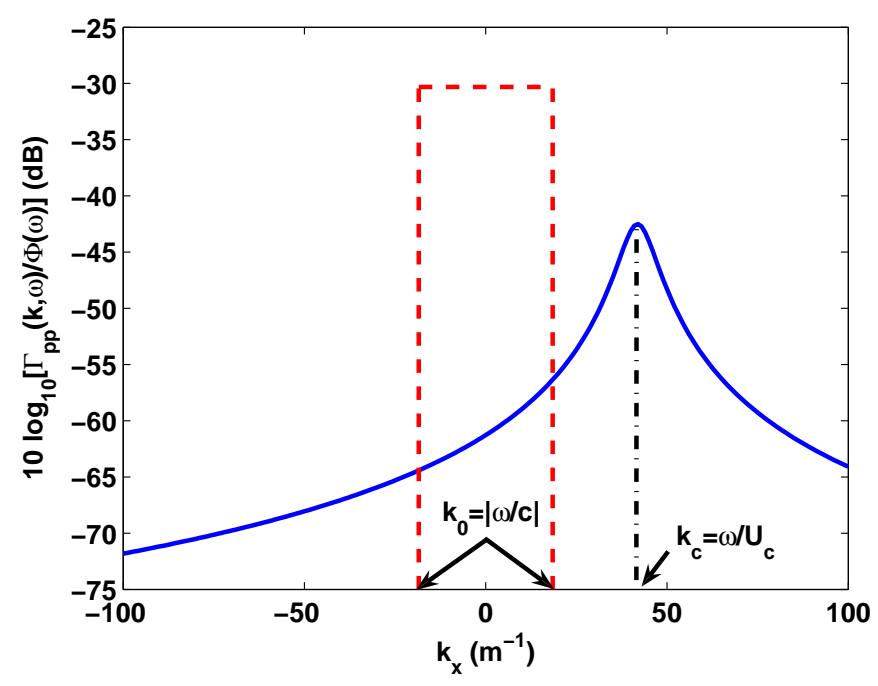

Fig. 1: Comparison of cross-spectral density function of (-) a TBL and (--) a diffuse field $-k_{c}$ : convective wavenumber, $k_{0}$ : acoustic wavenumber

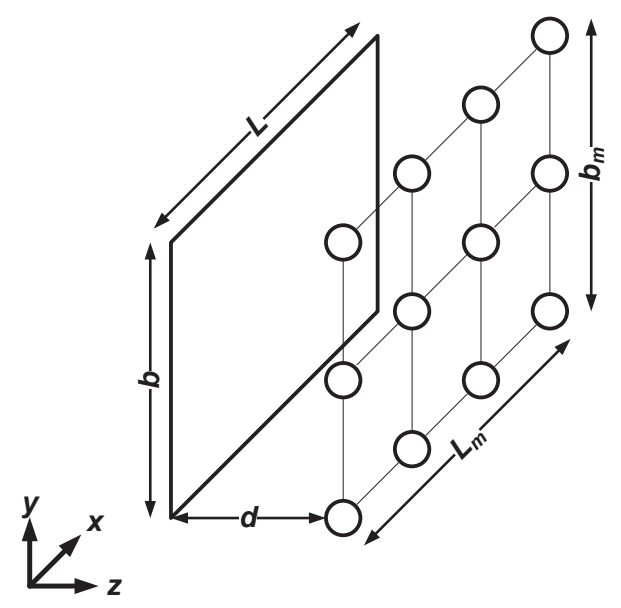

Fig. 2: Definition of the array of acoustic monopoles

To properly describe these interference conditions, one has to estimate, for each wall-pressure plane wave, the complex amplitude $B_{m}^{r s}(\omega)$ of each monopole $m$ of the array. Actually, this amplitude is obtained by writing the equality of a wall-pressure plane wave of unit amplitude and the wall-pressure field generated by the monopole array over a grid of $p$ observation points located on a rigid plane:

$$
\sum_{m} B_{m}^{r s}(\omega) H_{m p}(\omega)=\mathrm{e}^{j k_{r} x_{p}+j k_{s} y_{p}}
$$


where $H_{m p}(\omega)$ is the transfer function between a monopole $m$ of coordinates $\left(x_{m}, y_{m}, z_{m}\right)$ and an observation point $p$ of coordinates $\left(x_{p}, y_{p}, 0\right)$ belonging to a rigid wall.

However, it can be inferred from Eq. (7) that the more the values of the wavenumbers $k_{r}$ and $k_{s}$ are important, the more the number of monopoles necessary to properly reproduce a wall-pressure plane wave of unit amplitude is high. Consequently, using a monopole array can be intractable in practice. To bypass this experimental limitation, a Source Scanning Technique has be developed.

\subsection{Source Scanning Technique}

The Source Scanning Technique relies on the linearity of the problem and consists in using a single moving monopole-like source to reconstruct a target wall-pressure plane wave from sequential measurements. It could be stressed that it is closely related to the concept of synthetic antenna [15], which consists in post-processing the signals of a moving receiving array to reconstruct an unknown target image with a finer spatial resolution than that obtained with a fixed array. Actually, the reciprocal mechanism is used in this paper.

To properly implement this technique in the proposed experimental framework, one has to notice that the evaluation of the transfer function $H_{v}\left(Q, k_{r}, k_{s}, \omega\right)$ requires two measurements, the first one to characterize the acoustic medium and the second one to characterize the dynamic behaviour of the structure excited by an acoustic monopole. By combining both information, one can obtain an evaluation of the target transfer function, that can be used to compute the response of the structure to a TBL excitation. Consequently, the Source Scanning Technique is carried out in 3 steps.

\subsubsection{Step 1: Characterization of the source radiation in a real acoustic medium}

The characterization of the source radiation in a real acoustic medium is performed by the measurement of the transfer functions $H_{m p}(\omega)$, corresponding to the blocked pressure on a rigid wall at position $p$ due to the monopole-like source at position $m$ as illustrated in Fig. 3.

From this set of transfer functions, it is possible to compute the amplitude $B_{m}^{r s}(\omega)$ for each position of the monopole-like source and each couple of wavenumbers $\left(k_{r}, k_{s}\right)$ [see Eq. (7)]. 


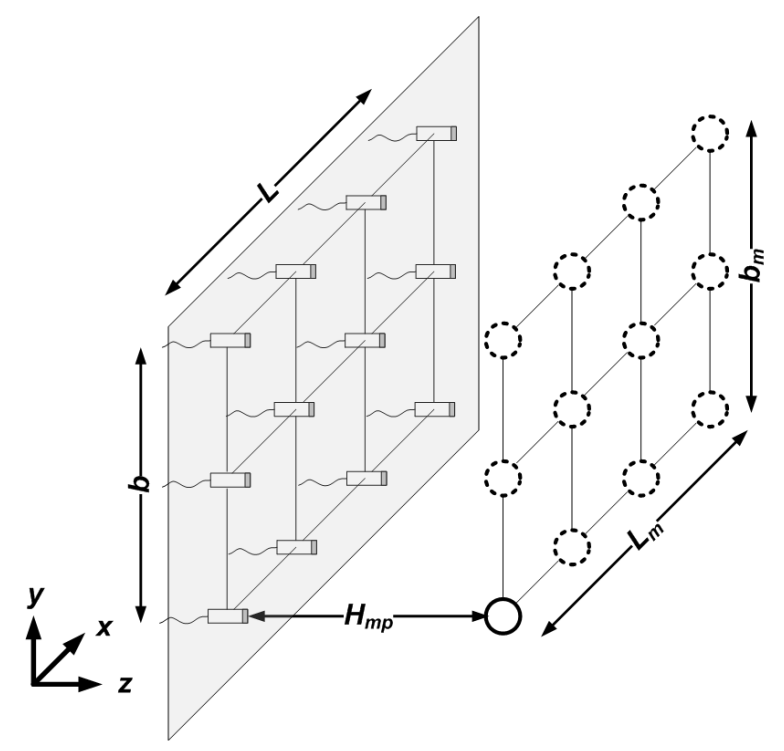

Fig. 3: Example of measurement of the transfer functions $H_{m p}(\omega)$

It should be noted that this step is compulsory if the acoustic medium can not be considered as semi-anechoic and the source as a monopole. However, it can be emphasized that the measurement have to be performed only once and are independent of the structure under test. On the contrary, if the acoustic medium is semi-anechoic and the source is a monopole, a theoretical expression of $H_{m p}(\omega)$ can be used to avoid these measurements. This expression writes:

$$
H_{m p}(\omega)=\frac{\mathrm{e}^{-j k_{0} \sqrt{\left(x_{m}-x_{p}\right)^{2}+\left(y_{m}-y_{p}\right)^{2}+z_{m}^{2}}}}{2 \pi \sqrt{\left(x_{m}-x_{p}\right)^{2}+\left(y_{m}-y_{p}\right)^{2}+z_{m}^{2}}} .
$$

\subsubsection{Step 2: Characterization of dynamic behaviour of the structure}

The characterization of the dynamic behaviour of the structure excited by an acoustic monopole is performed by the measurement of the transfer functions $H_{Q m}$, corresponding to the velocity at point $Q$ due to the monopole-like source at position $m$ [see Fig. 4].

From this set of transfer functions and the amplitude $B_{m}^{r s}(\omega)$ computed in the first step, the transfer function $H_{v}\left(Q, k_{r}, k_{s}, \omega\right)$ can be calculated off-line from the following relation:

$$
H_{v}\left(Q, k_{r}, k_{s}, \omega\right)=\sum_{m} H_{Q m}(\omega) B_{m}^{r s}(\omega)
$$




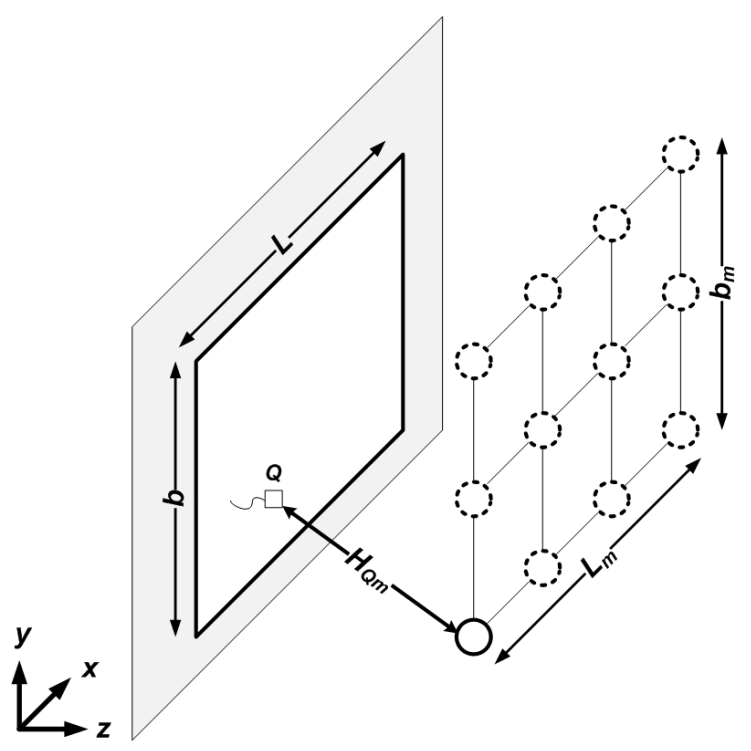

Fig. 4: Example of measurement of the transfer functions $H_{Q m}(\omega)$

\subsubsection{Step 3: Reconstruction of TBL-induced vibrations}

At this stage, the contribution of each wall-pressure plane wave are combined, by linear processing, to obtain the structural velocity ASD function from Eq. (6).

\subsubsection{Summary of SST}

In this section, SST is presented under a block diagram form to clearly distinguish measurement stages from numerical processing stages based on measured data [see Fig. 5].

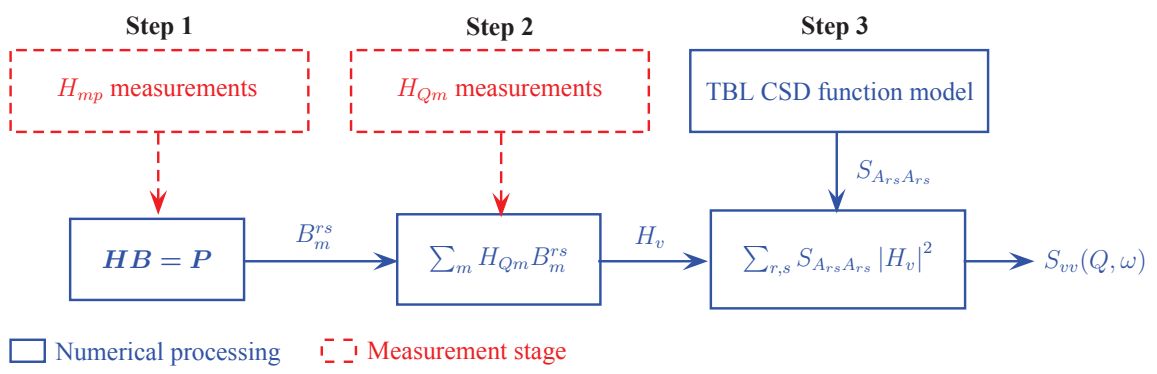

Fig. 5: Block diagram of the proposed experimental procedure 
The proposed block diagram shows that the use of a synthetic array requires two transfer functions measurements and numerical processings. Such a process presents several advantages to assess TBL-induced vibrations. Firstly, a synthetic array allows not only a greater flexibility with respect to the number of monopoles, but also to avoid the scattering of the sound field on a dense set of sources involved into a physical array. Furthermore, only transfer function measurements are required. These two latter points are very interesting, since it is not necessary to control the amplitude and phase of several sources simultaneously. Furthermore, dispersions of sensor characteristics are avoided as are all the reference problems occurring when an array of sources is used.

Nevertheless, the sequential nature of the experimental process requires measurements to be made with care. Indeed, inaccuracies of source positioning can generate phase shift dispersions between two successive positions of the source. This problem can be easily managed by using a two-axis robot. Another issue is related to the variations of the set-up with time, since sequential measurements are quite lengthy. Consequently, the behaviour of the structure may change during the experiment, because of environmental variations for instance. To insure the consistency of measurements throughout the experiment, measurements have to be made in a stable environment to keep the linearity assumption valid.

\section{Experimental aspects}

When analysing Eq. (6), it is obvious that the accuracy of the reconstruction of the structural velocity ASD function depends on two main criteria, namely the number of wall-pressure plane waves and the definition of the scanning grid.

\subsection{Number of wall-pressure plane waves}

\subsubsection{Basic principle}

The numerical evaluation of the series given by Eq. (6) theoretically requires an infinite number of wavenumber couples $\left(k_{r}, k_{s}\right)$. However, the structure acts as a wavevector filter characterized by the transfer function $H_{v}$. This enables limiting the wavenumber couples to those mainly contributing to the structural response. Consequently, the structural velocity ASD function can be approximated from a finite number of uncorrelated wall-pressure plane waves. This, however, requires some knowledge about the structure under test to determine an optimal cut-off wavenumber allowing properly truncating the series given by Eq. (6).

A simple indicator for the practical choice of $\bar{k}$ can be derived from the analysis of the physical mechanisms governing the response of a plate excited by a TBL, such as the aerodynamic coincidence and the filtering effect of the structure 
For a flat plate with natural bending wavenumber $k_{f}$, immersed in a light fluid such as air, the aerodynamic coincidence associated with the filtering effect of the structure on the TBL excitation allows explaining the physical mechanisms of the TBL-induced vibrations in a straightforward manner. In general, three configurations are observed. Indeed, for $k_{c}<k_{f}$, the resonant and non-resonant modes in aerodynamic coincidence of the plate are the main contributors to vibration response. For $k_{c} \approx k_{f}$, the number of resonant modes in aerodynamic coincidence is maximal [5]. In this case, these modes mainly contribute to the vibration response. Finally, for $k_{c}>k_{f}$, only the resonant modes have a significant contribution to the vibration response due to the filtering effect of the plate.

As a result, the wavenumbers $\left(k_{r}, k_{s}\right)$ defining the wall-pressure plane waves can be restrained to those matching with the modes, that mainly contribute to the vibration response. Furthermore, this analysis implies a substantial reduction in the number of positions of the monopole-like source, since only the plane waves matching with the most important modes have to be reconstructed. However, the a priori determination of structural modes as well as natural wavenumber $k_{f}$ can be a challenging task for industrial structures. For all these reasons, it is preferable to defined a simple but robust indicator to take into account all the physical mechanisms above-cited. Here, a practical choice of the cut-off wavenumber $\bar{k}$ is such that $\bar{k}>k_{f}$, where $k_{f}$ is the natural wavenumber of the plate equivalent to the real structure. Based on this indicator, the set of wall-pressure plane waves is thus defined to satisfy $\left(k_{r}, k_{s}\right) \in[-\bar{k}, \bar{k}]^{2}$ with a prescribed wavenumber resolution $\left(\Delta k_{r}, \Delta k_{s}\right)$. Of course, to take advantage of the filtering effect of the structure on the excitation, it is necessary to have a good knowledge of the structure under test. In particular, this means that for industrial structures, such as multilayer or ribbed structures, the dynamic behaviour has to be studied carefully to properly identify the cut-off wavenumber.

\subsubsection{Numerical validation}

To validate the reasoning presented in section 3.1.1, the response of a simply supported steel plate immersed in air and subjected to a TBL excitation model by a Corcos model [16] is calculated using either the classical random vibration theory or the wall-plane wave expansion.

For the sake of completeness, it should be noted that the reference calculation was carried out using the discretized version of the velocity ASD function given by the random vibration theory, that is:

$$
S_{v v}(Q, \omega)=\sum_{i, j} H_{v}^{*}\left(Q, M_{i}, \omega\right) S_{p p}\left(M_{i}, M_{j}, \omega\right) H_{v}\left(Q, M_{j}, \omega\right) \Delta M_{i} \Delta M_{j},
$$

where $S_{p p}\left(M_{i}, M_{j}, \omega\right)$ is the TBL CSD function, $\Delta M_{i}$ is the spatial resolution and $H_{v}\left(Q, M_{i}, \omega\right)$ is the transfer function corresponding to the structural velocity at point 
$Q$ when the plate is excited by a point force at point $M_{i}$.

In the present case, the transfer function $H_{v}\left(Q, M_{i}, \omega\right)$ is computed analytically using the following mode expansion:

$$
H_{v}\left(Q, M_{i}, \omega\right)=j \omega \sum_{n} \frac{\phi_{n}(Q) \phi_{n}\left(M_{i}\right)}{M_{n}\left(\omega_{n}^{2}-\omega^{2}+j \eta_{n} \omega \omega_{n}\right)},
$$

where $M_{n}$ is the generalized mass, $\omega_{n}$ the natural frequency of the plate, $\phi_{n}$ the mode shape, $\eta_{n}$ the modal damping.

Regarding the wall-plane wave expansion, the calculation of the velocity ASD function is derived from Eq. (6), in which the transfer function $H_{v}\left(Q, k_{r}, k_{s}, \omega\right)$ is written:

$$
H_{v}\left(Q, k_{r}, k_{s}, \omega\right)=j \omega \sum_{n} \frac{P_{n} \phi_{n}(Q)}{M_{n}\left(\omega_{n}^{2}-\omega^{2}+j \eta_{n} \omega \omega_{n}\right)},
$$

where the generalized force $P_{n}$ is given by the following equation:

$$
P_{n}=\int_{S_{p}} \mathrm{e}^{j k_{r} x+j k_{s} y} \phi_{n}(P) d S_{p} \approx \sum_{p} \mathrm{e}^{j k_{r} x_{p}+j k_{s} y_{p}} \phi_{n}^{p} \Delta S_{p},
$$

where $\phi_{n}^{p}$ is the mode shapes of the plate discretized over the p points and $\Delta S_{p}$ is a surface element. ble 1 .

The simulation parameters of the proposed numerical validation are given in Ta-

Table 1: Simulation parameters of the numerical validation

\begin{tabular}{ll}
\hline Parameters & Values \\
\hline Length of the plate & $0.6 \mathrm{~m}$ \\
Width of the plate & $0.3 \mathrm{~m}$ \\
Thickness of the plate & $5.10^{-3} \mathrm{~m}$ \\
Modal damping & $\eta_{n}=\eta=1 \%$ \\
Number of grid points & $231(21 \times 11)$ \\
Observation point & $Q(0.2 \mathrm{~m}, 0.1 \mathrm{~m})$ \\
Free stream velocity & $U_{\infty}=50 \mathrm{~m} . \mathrm{s}^{-1}$ \\
Longitudinal and lateral decay rates & $\left(\alpha_{x}, \alpha_{y}\right)=(0.116,0.7)$ \\
Maximal frequency studied & $f_{\max }=250 \mathrm{~Hz}$ \\
Cut-off wavenumber & $\bar{k}=50 \mathrm{~m} . \mathrm{s}^{-1}$ \\
Wavenumber resolution & $\Delta k_{r}=\Delta k_{s}=1 \mathrm{~m}^{-1}$ \\
\hline
\end{tabular}

The result presented in Fig. 6 shows a good agreement between the classical random vibration formulation and the wall-plane wave expansion and allows validating the selection process of the wall-plane waves explained in section 3.1.1. 


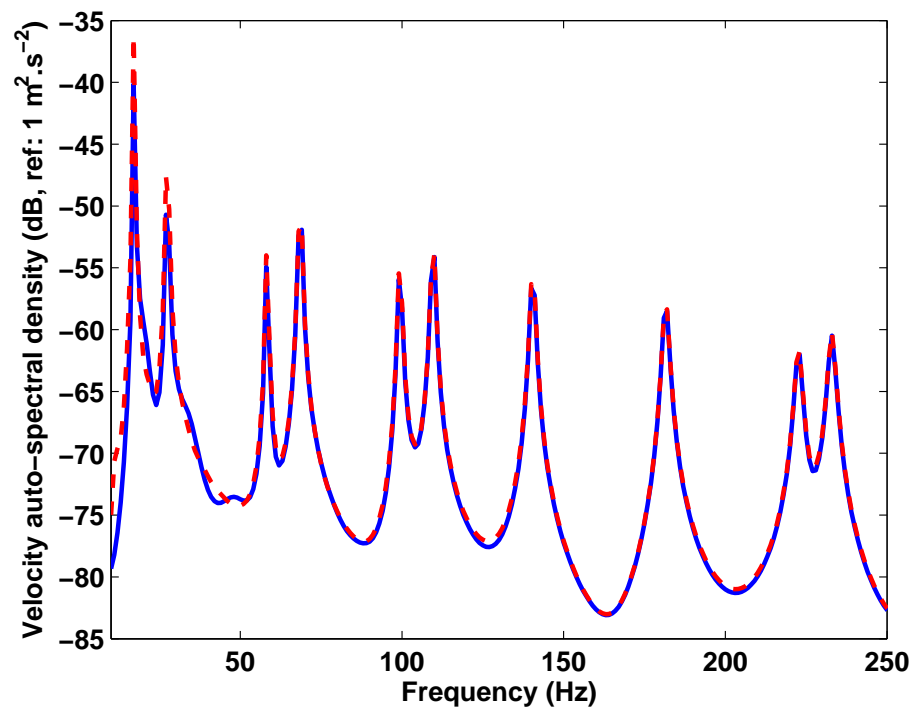

Fig. 6: Comparison of the structural velocity ASD function $S_{v v}(Q, \omega)$ at point $Q(0.2$ $\mathrm{m}, 0.1 \mathrm{~m}$ ) computed by (-) the classical random vibration formulation (Reference) and (--) the wall plane-wave expansion

\subsection{Definition of the scanning grid}

Three main parameters are involved in the design of the scanning grid, namely the number of grid points, its dimension and its distance from the observation area.

From a numerical parametric study using parameters defined in Table 1 [see Ref. [14] for further details], it has been shown that the grid covered by the monopole source has to satisfy the following criteria:

- a minimum of 4 monopoles per wavelength $\bar{\lambda}=\frac{2 \pi}{\bar{k}}$ is required to properly reconstruct a wall-pressure plane wave;

- the grid size has to be equal to or greater than the size of the observation area to ensure a good acoustic coverage of the observation area;

- the distance of the grid from the observation plane can be arbitrarily chosen in the interval $\left[\frac{\bar{\lambda}}{4}, 3 \bar{\lambda}\right]$ to limit the condition number of the propagation operator $H_{m p}$.

When applying all the rules cited above, the reconstruction of a wall-pressure plane wave as well as that of the TBL CSD function modelled by the Corcos model are very satisfactory as illustrated in Figs. 7 and 8.

It should be noted that this pre-design parametric study has been performed by considering theoretical acoustic monopoles. In practice, monopole-like sources dif- 


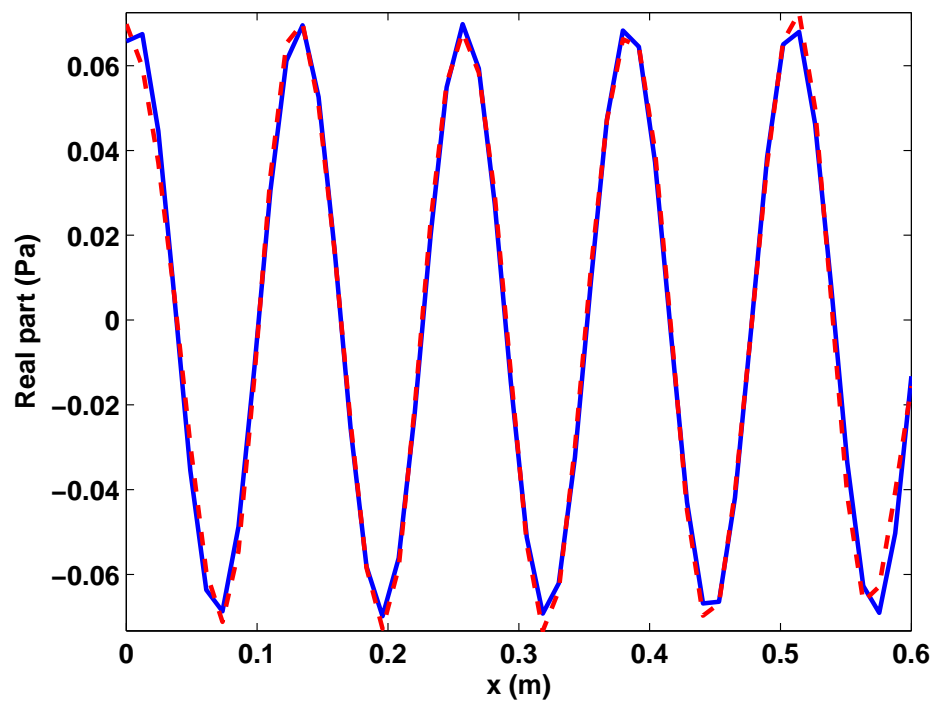

Fig. 7: Comparison between (-) the target wall-plane wave and (--) the reconstructed wall-pressure plane using the design rules of the scanning grid for $\left(k_{r}, k_{s}\right)=(\bar{k}, \bar{k})$ at $250 \mathrm{~Hz}$

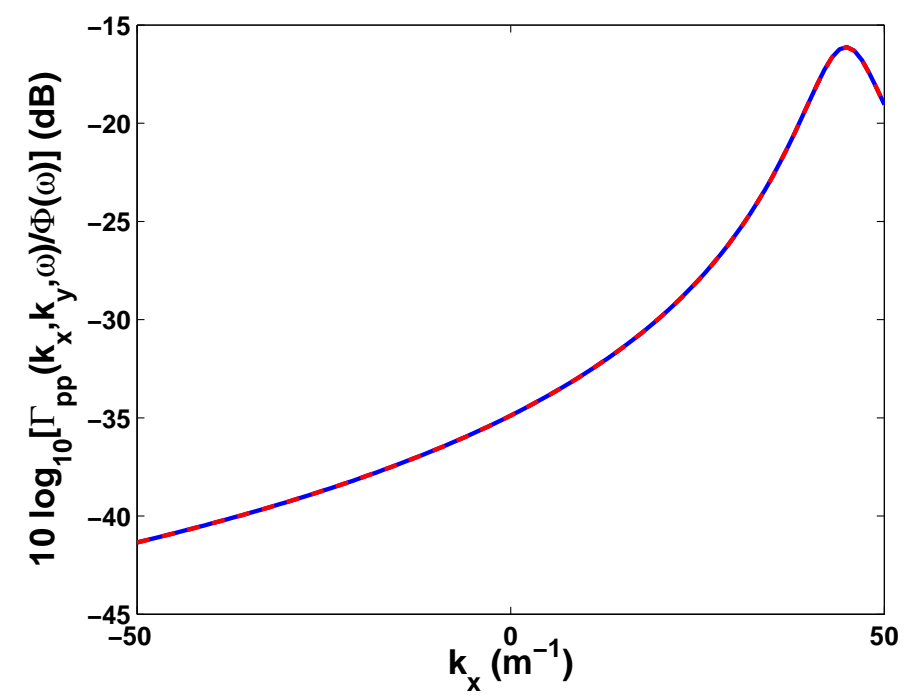

Fig. 8: Comparison between (-) the target TBL CSD function and (--) the reconstructed TBL CSD using the design rules of the scanning grid in the plane $\left(k_{x}, 0\right)$ at $250 \mathrm{~Hz}$ 
fer from theoretical ones, since the acoustic pressure field is not singular in the very near-field of the source. However, it has been pointed out that the source array should not be too close to ensure a good acoustic coverage of the observation area. Consequently, one can reasonably expect that above results remain acceptable for real acoustic monopole-like sources, as will be shown in section 4 .

\section{Experimental validation}

The aim of this experimental validation is to reproduce a wind measurement with the Source Scanning Technique. The wind tunnel measurement has been carried out by Totaro et al. [17] on a steel plate with dimensions $0.6 \times 0.3 \times 0.0005 \mathrm{~m}^{3}$ glued on its edges to a rigid baffle [see Fig. 9].

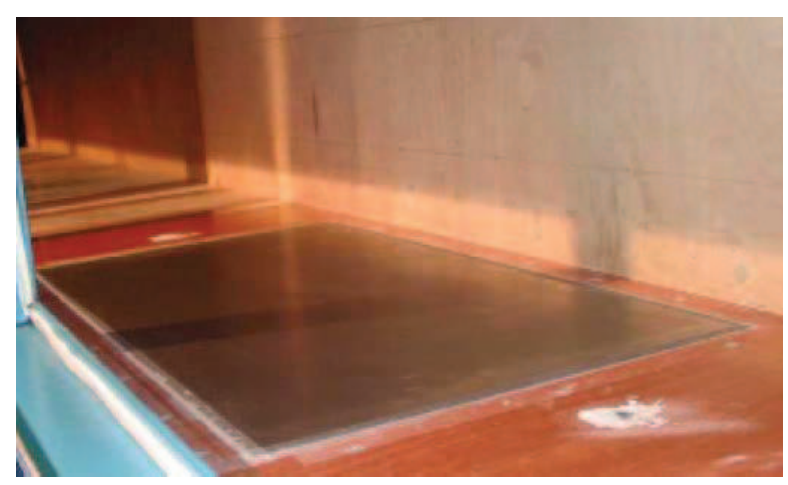

Fig. 9: Wind tunnel measurement

For this experiment, three quantities have been measured for a free stream velocity $U_{\infty}=50 \mathrm{~m} . \mathrm{s}^{-1}$, namely:

- the correlation parameters $\alpha_{1}=\frac{1}{\alpha_{x}}$ and $\alpha_{2}=\frac{1}{\alpha_{y}}$ of the Corcos model [see Fig. 10]:

- the TBL wall-pressure frequency spectrum $S_{p p}(\omega)$ required to scale the Corcos model [see Fig. 11]:

- the structural velocity ASD function at 72 points [see Fig. 12]:

\subsection{Description of the test bench}

As highlighted in section 2.4, SST requires the measurement of two sets of transfer functions, namely $H_{m p}$ and $H_{Q m}$, to derive the transfer function $H_{v}\left(Q, k_{r}, k_{s}, \omega\right)$ 


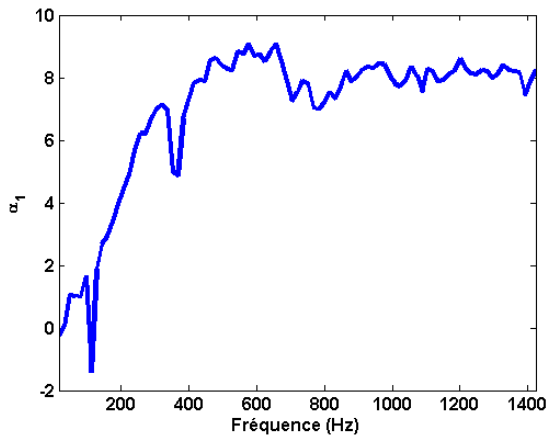

(a)

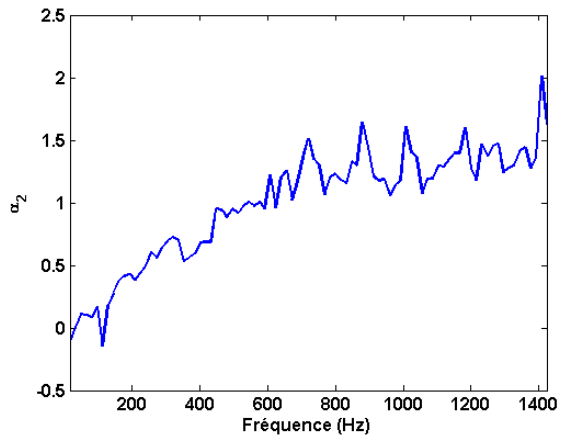

(b)

Fig. 10: Measurements of the the correlation parameters $\alpha_{x}$ and $\alpha_{y}$ of the Corcos model for $U_{\infty}=50 \mathrm{~m} \cdot \mathrm{s}^{-1}$ - (a) $\alpha_{1}$ and (b) $\alpha_{2}$, after Totaro et al. [17]

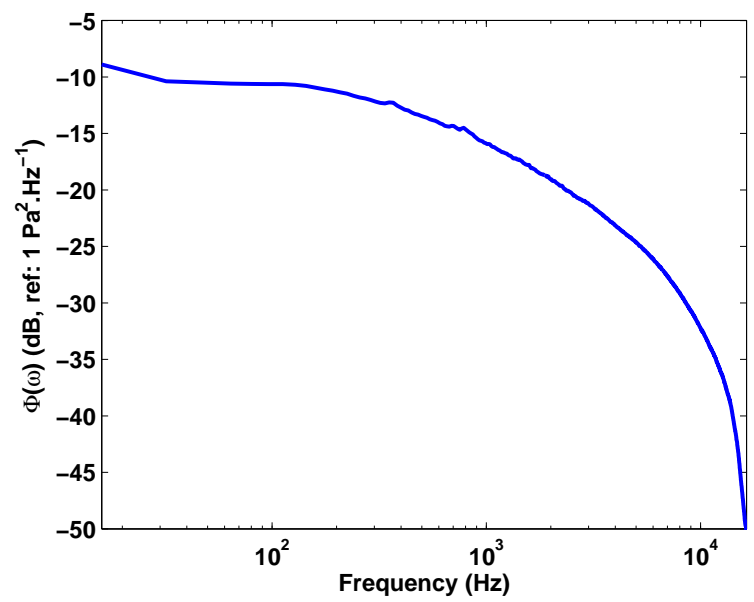

Fig. 11: TBL wall-pressure frequency spectrum measured in wind-tunnel for $U_{\infty}=$ $50 \mathrm{~m} \cdot \mathrm{s}^{-1}$, after Totaro et al. [17]

from Eq. (9). A schematic representation of the measurement process of $H_{m p}$ and $H_{Q m}$ has been proposed in Figs. 3 and 4.

From the practical point of view, the aim is to develop a test bench able to reproduce the measurement process of $H_{m p}$ and $H_{Q m}$ explained in section 2.4. 


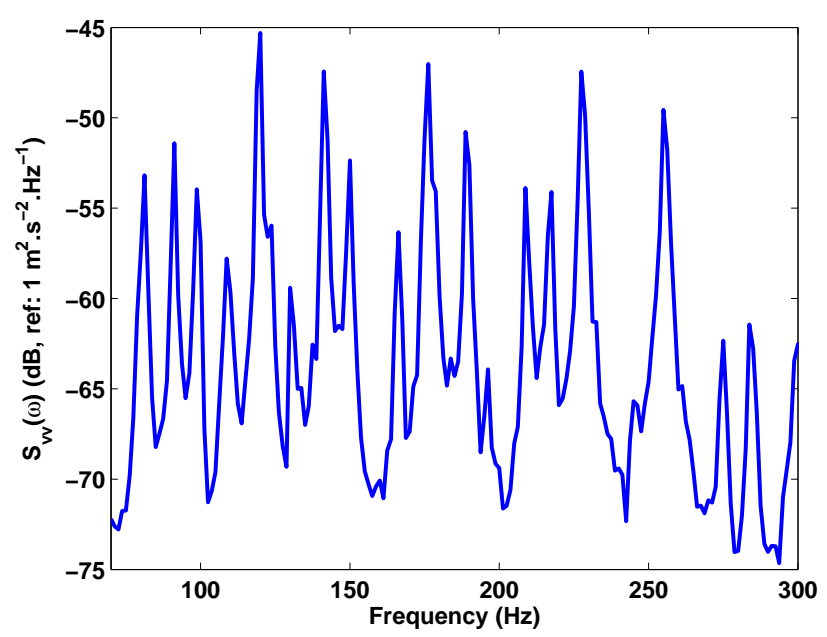

Fig. 12: Structural velocity ASD function at point $(x, y)=(0.22 \mathrm{~m}, 0.23 \mathrm{~m})$, after Totaro et al. [17]

\subsubsection{Experimental set-up for $\boldsymbol{H}_{m p}$ measurements}

The measurements of the transfer functions $H_{m p}$ between the particle velocity of the monopole-like source at positions $m$ and the pressure at positions $p$ have been carried out in a sound-treated room by using a white-noise excitation and measuring the blocked pressure on a rigid wall, consisting of a thick wooden plate $1.02 \times 1.25 \times 0.036 \mathrm{~m}^{3}$, as presented in Fig. 13 .

In this study, the excitation system consists in a monopole-like source, made with the help of a loudspeaker emitting noise through a tube, fixed to a two-axis robot, while the reception system is a near-field linear microphone array used to measure the blocked pressure over the rigid surface.

\subsubsection{Experimental set-up for $\boldsymbol{H}_{Q m}$ measurements}

The measurements of the transfer functions $H_{Q m}$ have consisted in measuring the transfer function between the particle velocity of the monopole-like source and the structural velocity at an observation point $Q$ located on the plate. To this end, an aperture of dimensions $0.6 \times 0.3 \mathrm{~m}^{2}$ has been made in the thick wooden plate used for $H_{m p}$ measurements, above which the studied plate was glued to allow bending motions. Then, the measurements of the structural velocity of the plate excited by the monopole source at positions $m$ have been performed with a lightweight 4 gram accelerometer to limit the effect of the added mass of the sensor on the plate. 


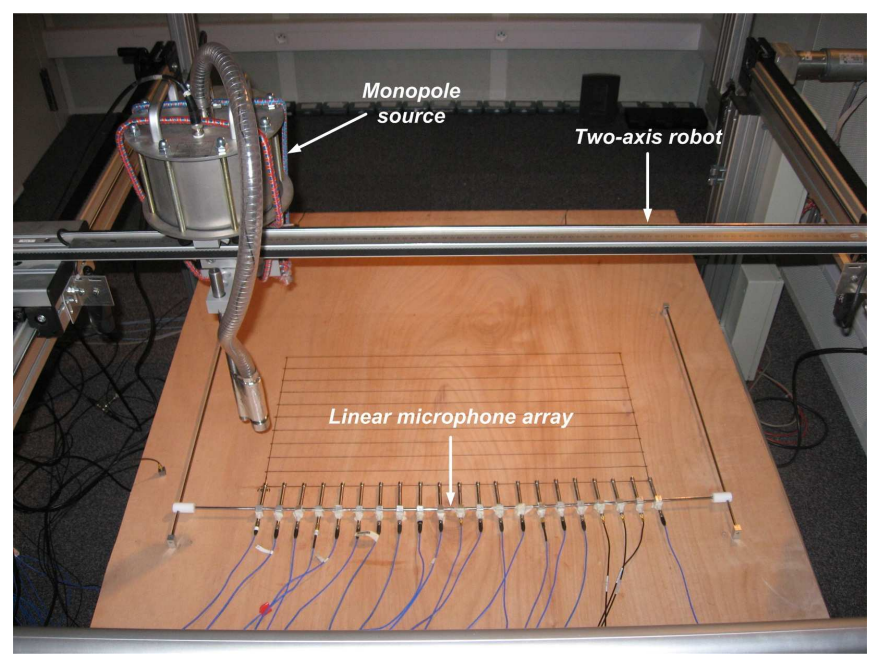

Fig. 13: Experimental set-up for measuring the transfer functions $H_{m p}$

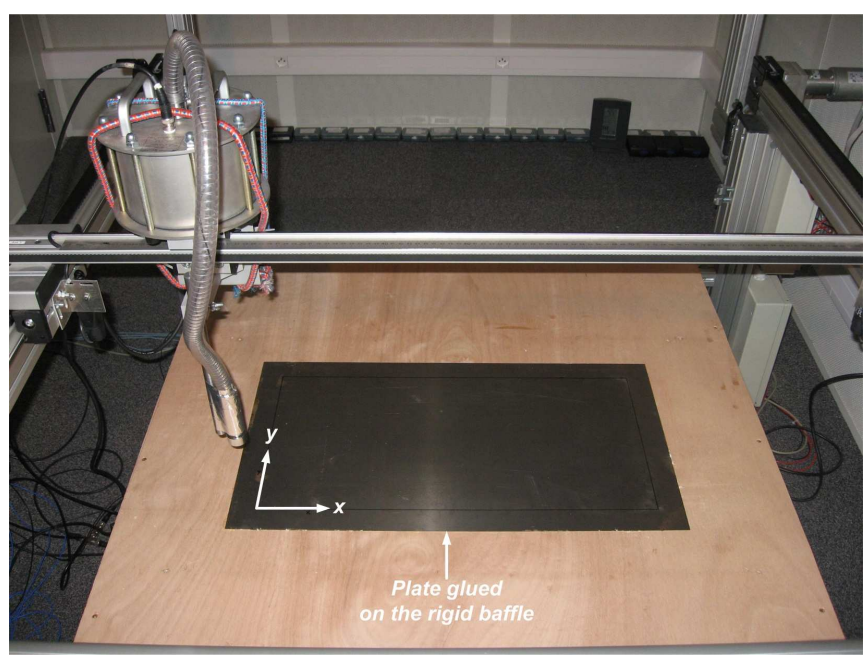

Fig. 14: Experimental set-up for measuring the transfer functions $H_{Q m}$ 


\subsection{Description of the monopole-like source}

As explained in section 4.1.2, the monopole-like source is obtained from a loudspeaker emitting a white noise excitation through a tube, whose diameter is smaller than a third of the acoustic wavelength [see Fig. 15]. The reference signal is measured by a velocity sensor fitted in a small nozzle mounted at the end of the tube, in order to take into account the standing waves existing in the tube. The inner diameter of the nozzle is $15 \mathrm{~mm}$. Consequently, the upper frequency for a monopole-like behaviour of the source is much lower than $7.5 \mathrm{kHz}$.

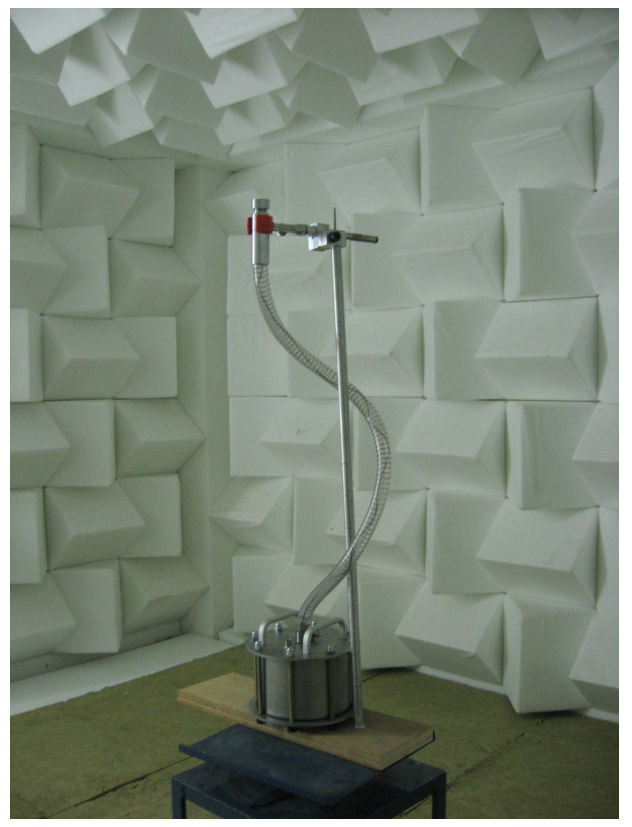

Fig. 15: Monopole-like source

To be considered as a monopole source, the physical source should have, in particular, an omnidirectional directivity pattern. This property of the source has been experimentally verified by measuring the acoustic pressure along two circumferences delimiting two orthogonal planes of a sphere of radius $30.5 \mathrm{~cm}$. These measurements are presented in Fig. 16.

Finally, the monopole source being not an efficient radiator at low frequency, the lower bound of the measurement frequency range is related to the signal-to-noise ratio. Preliminary measurements showed that SNR was acceptable for frequencies above $70 \mathrm{~Hz}$. 


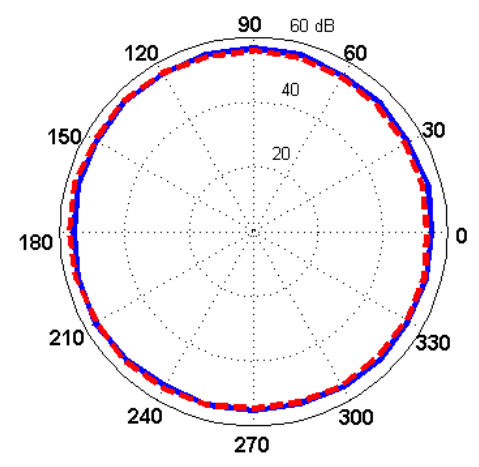

(a)

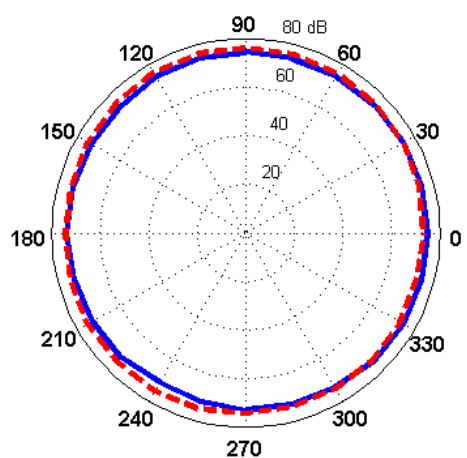

(b)

Fig. 16: Directivity pattern of the monopole-like source at (a) $125 \mathrm{~Hz}$ and (b) 2000 $\mathrm{Hz}-(-)$ First plane and (--) Second plane (perpendicular to the first plane)

\subsection{Study of measurement biases}

To properly analyse the experimental results presented in sections 4.4 and 4.6 , it is important to study the possible measurement biases beforehand.

\subsubsection{Screen effect of the baffle}

As shown in Figs. 13 and 14, the dimensions of the baffle are finite. As a result, a part of the pressure emitted by the source on the upper face of the plate can be indirectly transmitted to the other face due to the diffraction of the pressure field on the edges of the baffle. That is why, it is worth ensuring that the pressure field measured on the hidden face of the plate is weak compared to the the one measured on the upper face in order to reproduce as accurately as possible the wind tunnel measurement. For this purpose, the pressure is measured on both sides of the baffle as illustrated in Fig. 17.

From there, an indicator $E$ is defined as the difference of the pressure levels measured on both sides of the baffle, namely:

$$
E=20 \log _{10}\left(\frac{\left|p_{u}\right|}{\left|p_{h}\right|}\right)
$$

where $p_{u}$ and $p_{h}$ are respectively the acoustic pressure measured on the upper and hidden faces of the baffle.

Fig. 18 presents the value of the indicator $E$ with respect to frequency. It shows that the pressure on the hidden face of the baffle is at least $10 \mathrm{~dB}$ lower than the 


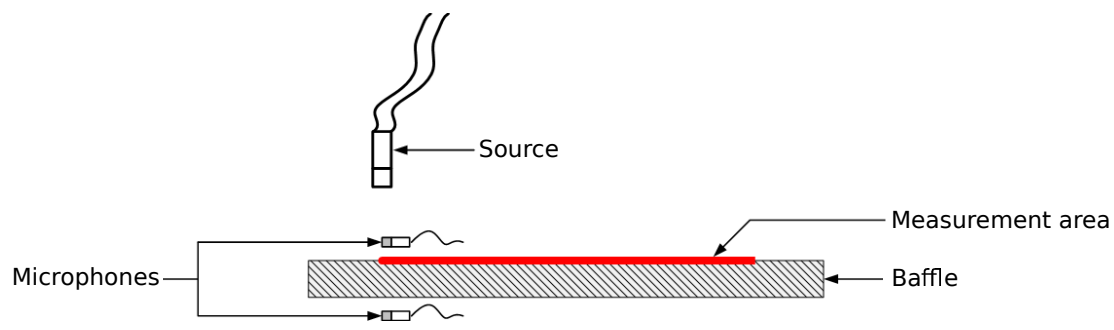

Fig. 17: Measurement of the screen effect of the baffle

pressure measured on the upper face of the baffle. Consequently, the bias introduced by the finite dimensions of the baffle is negligible.

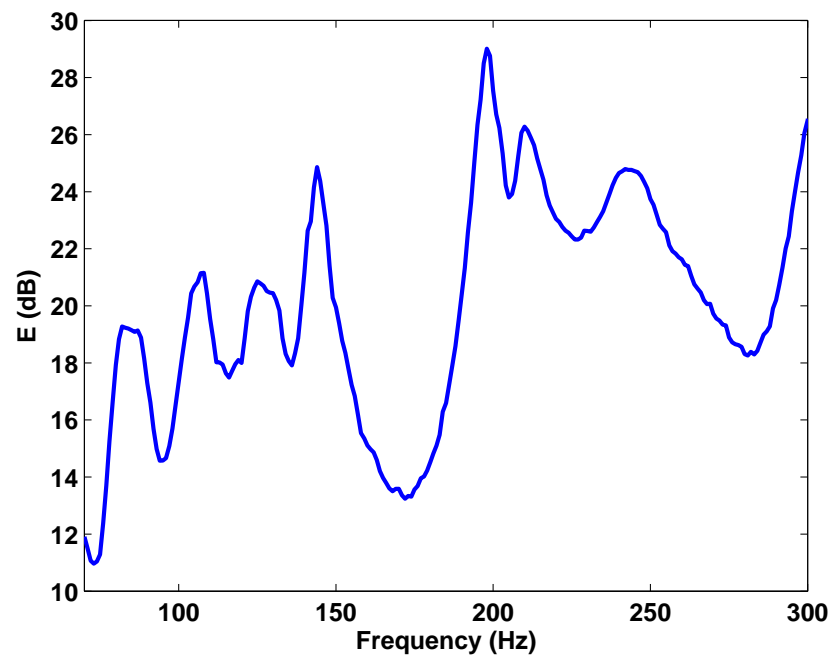

Fig. 18: Screen effect of the baffle

\subsubsection{Baffle rigidity}

The rigidity of the baffle is an important parameter to ensure the validity of $H_{Q m}$ measurements. Indeed, if the baffle and plate vibration levels are of the same order, then the boundary conditions of the plate will be altered. In the present paper, the baffle rigidity is evaluated by measuring the difference of the vibration levels at a point $A_{1}(0.22 \mathrm{~m}, 0.23 \mathrm{~m})$ of the plate and a point $B_{1}(-0.08 \mathrm{~m}, 0.675 \mathrm{~m})$ of the baffle (The coordinates of each point are defined in the frame of the plate presented in Fig. 4). Practically, vibration levels are measured using accelerometers. From 
there, an indicator $E_{v}$, defining the difference of the vibration levels, is derived as in Eq. (14).

Fig. 19 presents the value of the indicator $E_{v}$ with respect to frequency. This figure shows that the difference of the vibration levels ranges from 5 to $10 \mathrm{~dB}$ in average. Consequently, baffle vibrations are larger than expected. The direct consequence is an alteration of the boundary conditions of the plate since vibration energy is injected to the structure through the baffle. This can be a major bias when comparing SST with wind tunnel measurements.

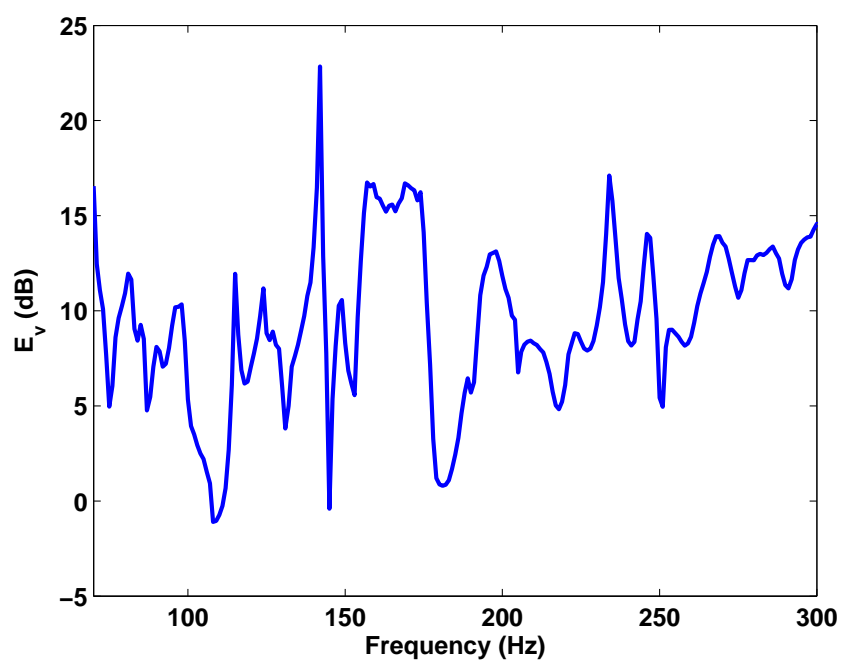

Fig. 19: Influence of the baffle rigidity

\subsubsection{Influence of the accelerometers}

The thickness of the plate under test being relatively low, the accelerometers used to measure the vibration field can modify its dynamic behaviour. Practically, only one accelerometer has been used. To assess the influence of this accelerometer on the dynamic behaviour of the plate, the input mobility $Y_{p}$ of the studied plate equipped with a lightweight accelerometer (4 grams) has been numerically computed from the following formula [26]:

$$
Y_{p}=\widetilde{Y}_{p} \frac{Y_{a}}{Y_{a}+\widetilde{Y}_{p}}
$$

where $\widetilde{Y}_{p}$ is the input mobility of the plate alone and $Y_{m}$ is the mobility of the accelerometer of mass $m$. 
Eq. (15) indicates that the eigen frequencies of the plate are modified by the presence of the accelerometer. The modified eigen frequencies can be obtained from the following equation:

$$
\operatorname{Im}\left(\widetilde{Y}_{p}\right)=\operatorname{Im}\left(Y_{a}\right)=\frac{1}{m \omega},
$$

where $\operatorname{Im}\left(\widetilde{Y}_{p}\right)$ is the imaginary part of $\widetilde{Y}_{p}$.

A graphical representation of the previous equation is proposed in Fig. 20. In particular, it shows that if the structure is lightly damped (which is the case here), then the presence of a sole accelerometer on the structure has almost no influence on the dynamic behaviour of the plate. As a consequence, the use of an unique accelerometer glued on the plate does not disturb the measurement of the transfer functions $H_{Q m}$.

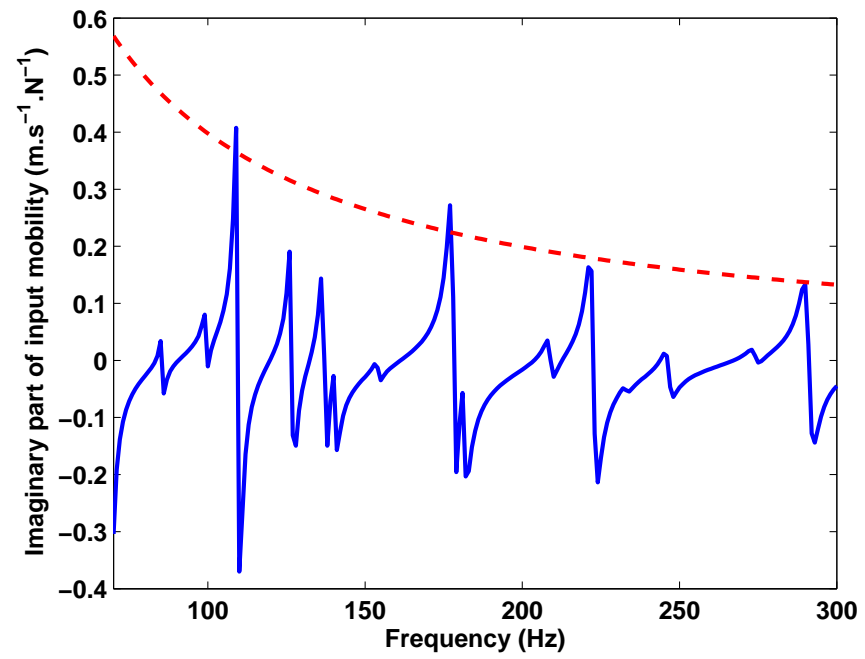

Fig. 20: Influence of the accelerometer mass on the eigen frequencies of the plate (-) Imaginary part of the input mobility $\widetilde{Y}_{p}$ of the plate alone and (--) Imaginary part of the acceleroter's mobbility $Y_{a}$

\subsection{Reconstruction of wall-pressure plane waves}

In this section, a wall-pressure plane wave of unit amplitude is compared to that reconstructed by using SST for two couples of wavenumbers $\left(k_{r}, k_{s}\right)=\left(4.5 \mathrm{~m}^{-1}, 4.5 \mathrm{~m}^{-1}\right)$ and $\left(k_{r}, k_{s}\right)=\left(20 \mathrm{~m}^{-1}, 20 \mathrm{~m}^{-1}\right)$. 
Fig. 21 presents, for both couples of wavenumbers, the comparison of the wallpressure plane waves to be reconstructed and those reconstructed from SST at 250 $\mathrm{Hz}$. This figure shows clearly that the amplitudes as well as the spatial variations of the wall-pressure plane waves are well reproduced. Consequently, this validates the experimental technique proposed for reconstructing wall-pressure plane waves.

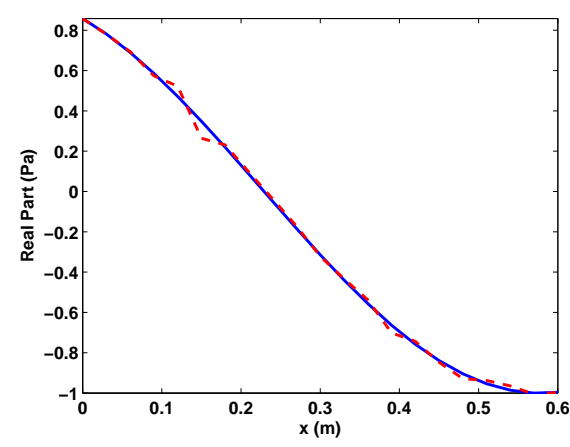

(a)

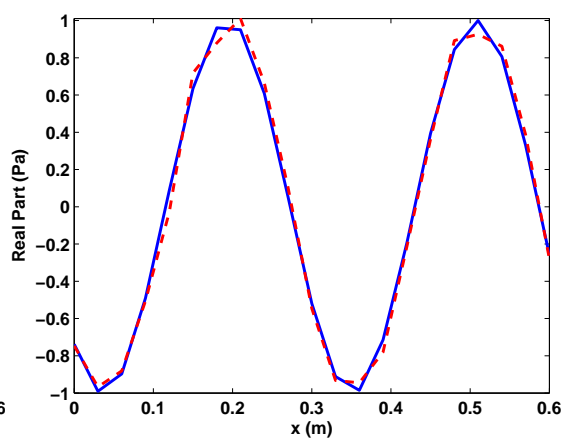

(b)

Fig. 21: Reconstruction of wall-pressure plane waves (a) for $\left(k_{r}, k_{s}\right)=$ $\left(4.5 \mathrm{~m}^{-1}, 4.5 \mathrm{~m}^{-1}\right)$ and $(\mathrm{b})$ for $\left(k_{r}, k_{s}\right)=\left(20 \mathrm{~m}^{-1}, 20 \mathrm{~m}^{-1}\right)$ in the plane $(x, 0.12 \mathrm{~m})$ at $250 \mathrm{~Hz}-(-)$ wall-pressure plane wave to be reconstructed (Reference) and (--) wall-pressure plane wave reconstructed by SST

\subsection{Semi-analytical validation of SST}

In this section, a semi-analytical validation of SST is proposed. It consists in comparing the structural velocity ASD function at one point of simply supported plate calculated on the basis of the classical random vibration formulation given by Eq. (10) with that obtained from SST. The proposed validation is semi-analytical since the transfer functions $H_{m p}$ are measured while the transfer functions $H_{Q m}$ are computed using the following relation:

$$
H_{Q m}(\omega)=j \omega \sum_{n} \frac{P_{n}^{m} \phi_{n}(Q)}{M_{n}\left(\omega_{n}^{2}-\omega^{2}+j \eta_{n} \omega \omega_{n}\right)},
$$

where the generalized force $P_{n}^{m}$ is written:

$$
P_{n}^{m} \approx \sum_{p} H_{m p}(\omega) \phi_{n}^{p} \Delta S_{p}
$$


Fig. 22 presents a comparison of the structural velocity ASD function at point $\mathrm{Q}$ $(0.22 \mathrm{~m}, 0.23 \mathrm{~m})$ of a $0.6 \times 0.3 \times 0.0005 \mathrm{~m}^{3}$ simply supported steel plate, obtained either from the classical random vibration formulation given by Eq. (10) or assessed by SST from Eqs. (6) and (9). As expected, good agreement between both results is observed, despite discrepancies of $3 \mathrm{~dB}$ in some frequency bands of low level, which is not crucial, since the vibration levels at the resonance frequencies are well estimated. Thus this result allows validating the experimental technique developed in this paper to characterize the vibrations induced by TBL.

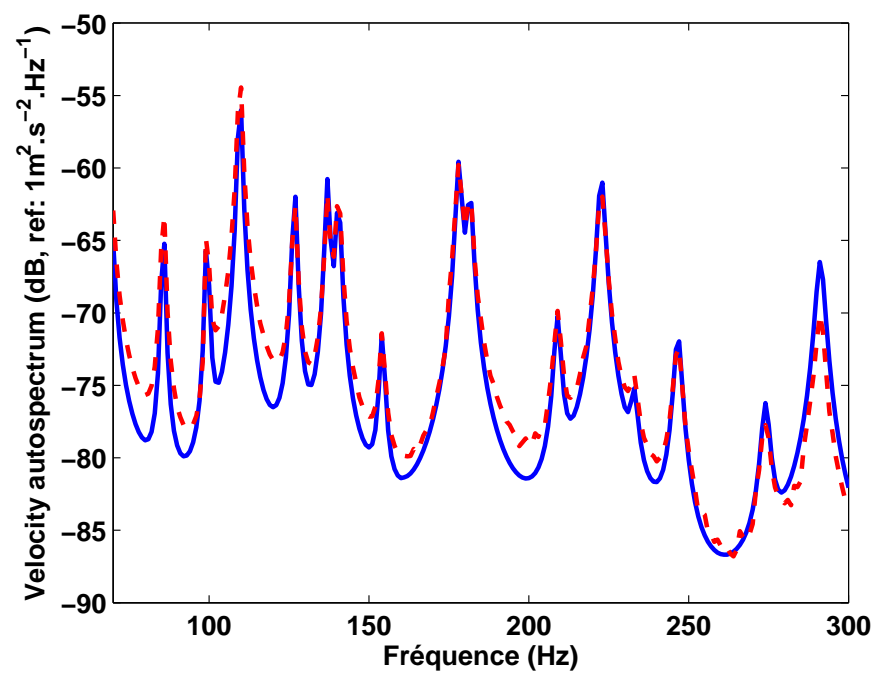

Fig. 22: Comparison of the structural velocity auto-spectral density function $S_{v v}(Q, \omega)$ at point Q $(0.22 \mathrm{~m}, 0.23 \mathrm{~m})$ computed by $(-)$ the classical random vibration formulation (Reference) and (--) SST by using the measurements of $H_{m p}$ and a semi-analytical calculation of $H_{Q m}$

\subsection{Complete experimental validation}

In the present section a complete experimental validation is proposed. This means that the measured transfer functions $H_{m p}$ and $H_{Q m}$ have been used to simulate the vibration response at one point of the plate from Eqs. (6)-(9). Considering the measurement biases detailed in section 4.3, discrepancies between SST results and wind tunnel measurements are expected. 
This is confirmed by Fig. 23 presenting a comparison of structural velocity ASD function at point $A_{1}$ measured in wind tunnel or reconstructed from SST. This comparison shows that the frequency content of the reconstructed ASD function differs from the measured one, while the range of variation of the ASD function is well described. The observed frequency discrepancy is mainly explained by the rigidity of the baffle which leads to an alteration of the boundary conditions as underlined in section 4.3.2.

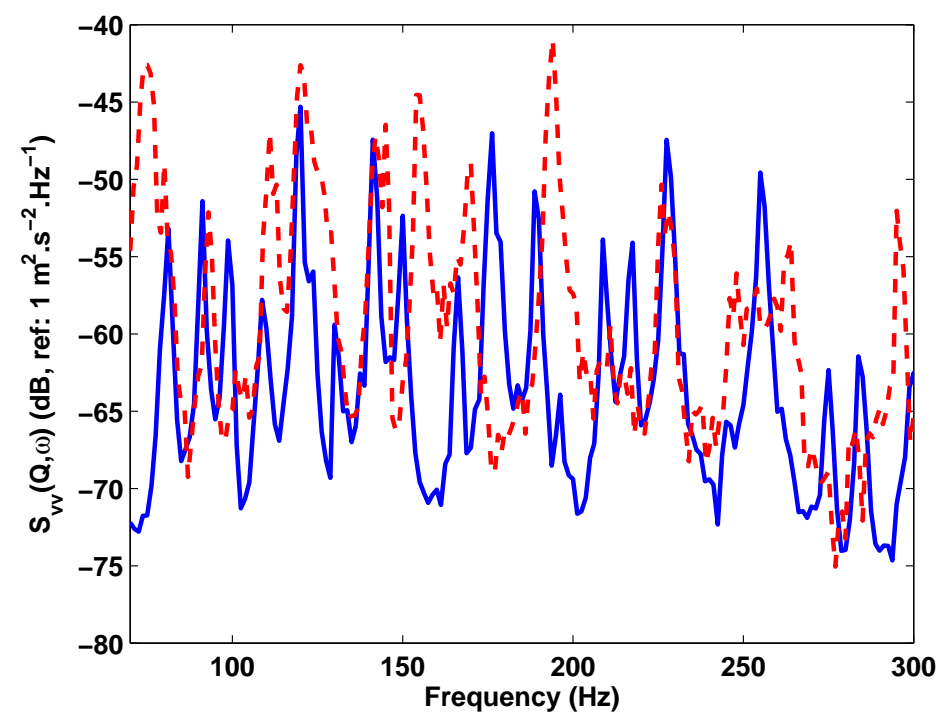

Fig. 23: Comparison of the structural velocity auto-spectral density function $S_{v v}(Q, \omega)$ at point Q $(0.22 \mathrm{~m}, 0.23 \mathrm{~m})(-)$ measured in wind tunnel and (--) reconstructed using SST

\subsection{Versatility of SST}

Numerous models are available in the literature to represent TBL wall-pressure fluctuations on a rigid plane. These models link wall-pressure fluctuations to the main features of the flow (convection velocity, boundary layer thickness, ...). One of the main features of SST is its versatility with respect to the TBL excitation model $\Gamma_{p p}\left(k_{r}, k_{s}, \omega\right)$, since the technique proposed is based on discrete wave-vector integration models. Consequently, all the TBL models expressed in the wavenumberfrequency space can be used. Furthermore, the comparison of these models can be carried out in a straightforward manner, since the introduction of TBL excitation is 
performed in the numerical stages by using Eqs. (6) and (9).

To demonstrate the ability of our experimental technique to deal with different TBL excitation models, the full implementation of SST is adopted, that is to say that measurements of $H_{m p}$ and $H_{Q m}$ are used to evaluate the TBL-induced vibrations of the steel plate defined previously.

In the following discussion, TBL models proposed by Corcos [16], Efimtsov [18], Chase [19] and Smol'yakov and Tkachenko [20] are compared for a fully developed turbulent flow whose parameters were measured in a wind tunnel by Totaro et al. [17] (see table 2). Fig. 24 presents the wavenumber-frequency spectrum predicted by the 4 models. For the sake of brevity, these models are not presented here, but detailed reviews and discussions about TBL models can be found in Refs. [21], [22] and [23]. Finally, the parameters of the TBL models used in this article are those proposed by their authors, except for the Corcos model, whose parameters are defined from measurements presented in Fig. 10.

Table 2: Turbulent flow parameters after Totaro et al. [17]

\begin{tabular}{ll}
\hline Parameters & Values \\
\hline Convection velocity & $U_{c}=35 \mathrm{~m} \cdot \mathrm{s}^{-1}$ \\
Friction velocity & $u_{\tau}=1.96 \mathrm{~m} \cdot \mathrm{s}^{-1}$ \\
Boundary layer thickness $\delta=85 \mathrm{~mm}$ \\
Displacement thickness & $\delta^{*}=8.8 \mathrm{~mm}$ \\
Momentum thickness & $\theta=6.7 \mathrm{~mm}$ \\
Wall shear stress & $\tau_{w}=4.58 \mathrm{~Pa}$ \\
\hline
\end{tabular}

The comparison of the structural velocity ASD function $S_{v v}(Q, \omega)$ at point Q $(0.22 \mathrm{~m}, 0.23 \mathrm{~m})$ of the plate, evaluated from SST for the four TBL excitation models mentioned above, is presented in Fig. 25. Observation of these results brings to light the fact that they are clustered together within $6 \mathrm{~dB}$ at most throughout the frequency range, despite large differences of TBL wall-pressure spectra in the lowwavenumber region. These results are consistent with previous investigations into TBL-induced vibrations [23, 24, 25]. Indeed, plate bending waves are only excited by the TBL pressure components of the matching scales and, in the present case, the bending waves are predominantly excited by convective components, since the frequency range of interest is below the aerodynamic coincidence frequency $(\approx$ $250 \mathrm{~Hz}$ ) for the plate under consideration. Consequently, the differences observed in Fig. 25 can be explained for the most part by the modelling of the convective components in the four TBL models, as shown in Fig. 24. 


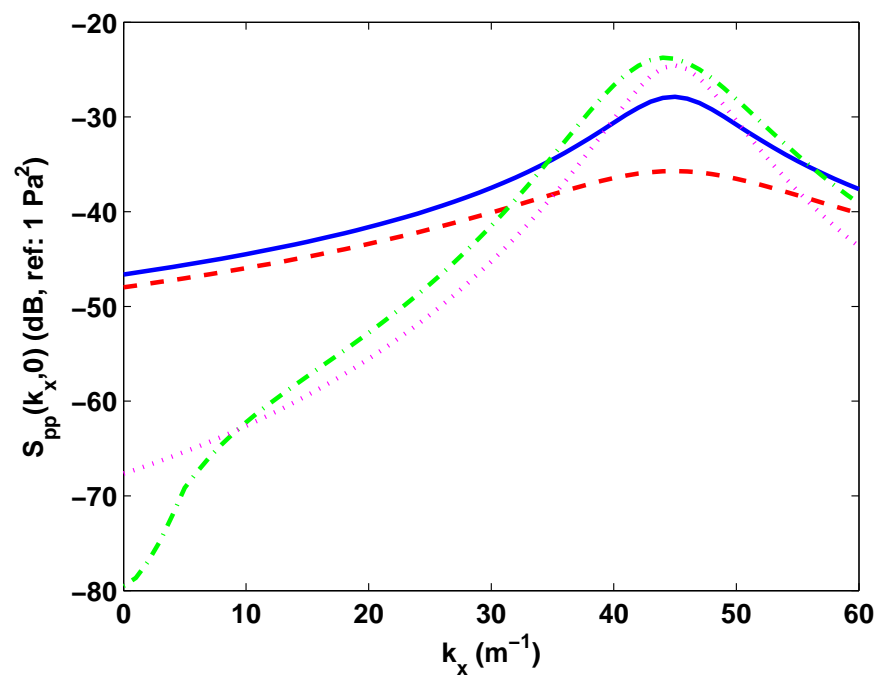

Fig. 24: Comparison of TBL wavenumber-frequency spectra at $250 \mathrm{~Hz}-(-)$ Corcos model, (--) Efimtsov model, (-., Chase model and (...) Smol'yakov \& Tkachenko model

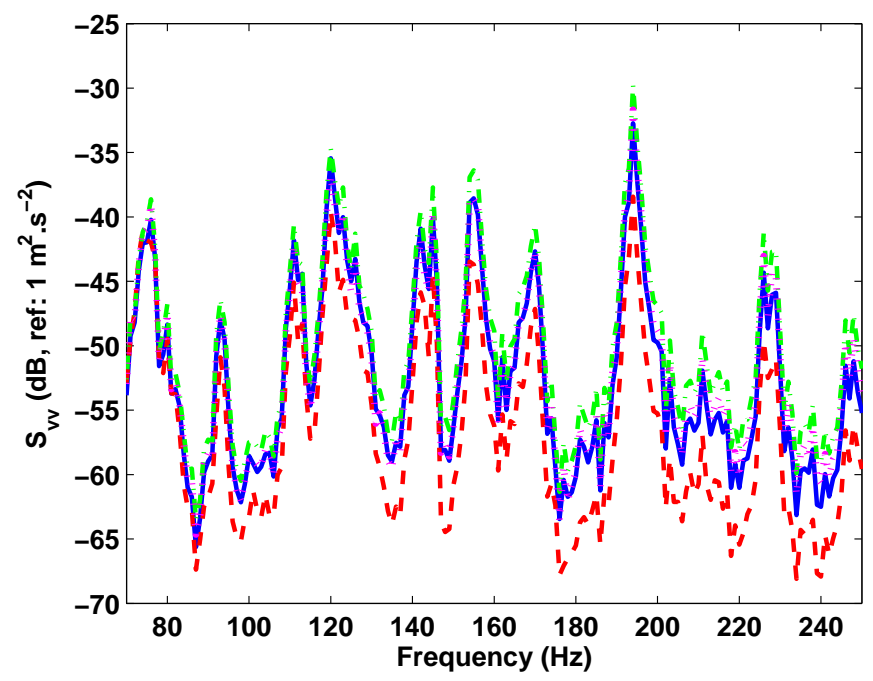

Fig. 25: Comparison of structural velocity auto-spectral density function $S_{v v}(Q, \omega)$ at point $\mathrm{Q}(0.22 \mathrm{~m}, 0.23 \mathrm{~m})$ for various TBL excitation models - (-) Corcos model, (--) Efimtsov model, (-., ) Chase model and (...) Smol'yakov \& Tkachenko model 


\section{Possible implementation of SST in an industrial context}

The test bench presented in section 4 has been developed for validation purposes only and can not be used as it is in an industrial context. For industrial applications, a test bench could be developed for simulating the vibro-acoustic behaviour of multilayer structures submitted to a random excitation in a controlled environment.

As illustrated in Fig. 26, the proposed test bench is made up of two rooms separated by a concrete wall, in which an aperture is made for inserting the structure under test. The relatively small size of the synthetic array allows installing in receiving and emission rooms climatic chambers for controlling temperature (typically in the range of $-30^{\circ} \mathrm{C}$ to $+70^{\circ} \mathrm{C}$ ) and humidity (standard conditions). In this context, the size of the excitation device is one of the advantage of SST compared to reverberant rooms or wind tunnel for which temperature and humidity control can not be performed at low cost.

To properly perform acoustic transparency measurements, the receiving room should be anechoic, while the emission room should be sound-treated. This experimental facility allows performing two types of research:

- Studying the filtering effect of multilayer structures on the TBL excitation. Indeed, for industrial multilayer structures, the filtering effect is not well controlled, while it could be a means of designing such a structure to noise annoyance due TBL excitation. In this context, the test bench described above would help in analysing experimentally the filtering effect of the structure by studying the vibro-acoustic response of the structure to wall-pressure plane waves generated by the synthetic array;

- Studying the influence of climatic conditions on the transmission loss of structures subjected to random excitations. Such studies are relevant in an industrial context, since mechanical properties of numerous materials used in industry (visco-elastic materials, rubber, etc.) depend on the temperature and hygrometric parameters. The variations of mechanical parameters with respect to environmental conditions play a central role in the vibro-acoustic behaviour of structures. Howerver, because of the practical difficulty of maintaining temperature and humidity levels in a reverberant room or in a wind tunnel, the influence of environmental parameters is often neglected. From this particular standpoint, the proposed test bench would be an original experimental facility.

\section{Conclusion}

The Source Scanning Technique aims at simulating experimentally the response of structures excited by stationary random pressure fields, such as TBL excitation. It relies on the experimental simulation of a set of uncorrelated wall-pressure plane waves, whose amplitudes are scaled on the TBL CSD function expressed in the 


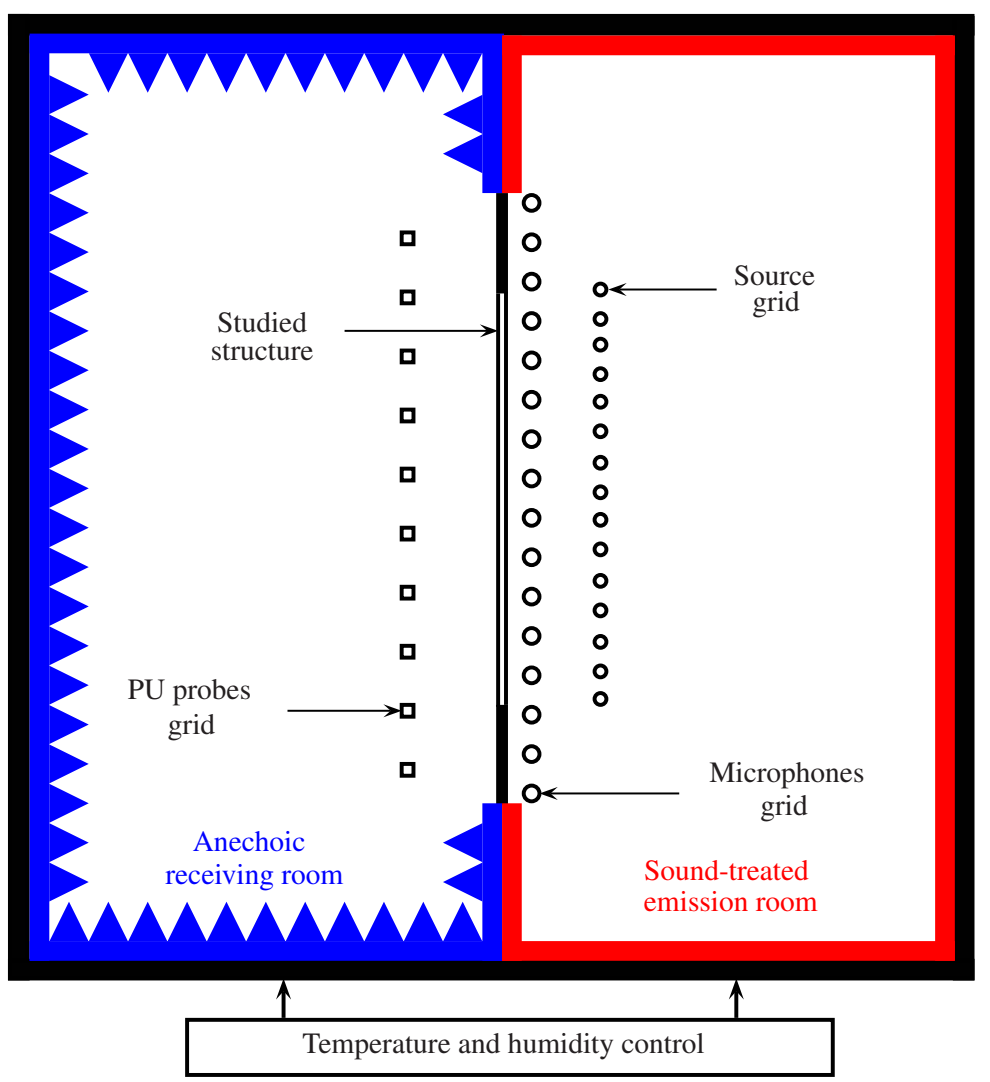

Fig. 26: Schematic representation of the proposed test bench

wavenumber space. From the practical point of view, the set of wall-pressure plane waves can be reconstructed using an array of acoustic monopoles. Nevertheless, such an approach can be intractable in practice because of the increase in the sources density with frequency. The key point of the proposed technique is the use of a synthetic array made up of a single acoustic monopole moved in space to replace the monopole array. By virtue of its sequential nature, SST allows a higher flexibility of the experimental set-up regarding the total number of monopoles required to suitably reconstruct wall-pressure plane waves. The counterpart of this sequential nature is the need of a precise source positioning to avoid phase shifts, as well as the need of a stable environment to avoid variations of the experimental set-up with time.

From these technical requirements, an experimental set-up was designed to validate the approach proposed under laboratory conditions. The results obtained on an academic structure show the method is capable of reconstructing wall-pressure plane waves and the structural velocity ASD function of a plate subjected to TBL excita- 
tion. Finally, the versatility of SST with respect to TBL excitation models has been highlighted.

In the future, SST could be used to develop a test bench for simulating the vibroacoustic behaviour of multilayer structures submitted to a random excitation (e.g. diffuse sound field or TBL excitations) in a controlled environment (i.e. humidity and temperature).

\section{References}

1. Bhat, W.V.: Flight test measurement of exterior turbulent boundary pressure fluctuations on Boeing 737 airplane. Journal of Sound and Vibration 14 (4), 439-457 (1971)

2. Galib, T.A., Katz, R.A., Ko, S.H., Sandman, B.: Measurements of turbulent pressure fluctuations using a buoyant vehicle coated with a thin elastomer layer. Journal of the Acoustical Society of America 96 (6), 3800-3803 (1994)

3. Finnveden, S., Birgersson, F, Ross, U., Kremer, T.: A model of wall pressure correlation for prediction of turbulence-induced vibration. Journal of Fluids and Structures 20, 1127-1143 (2005)

4. Fahy, F.: On simulating the transmission through structures of noise from turbulent boundary layer pressure fluctuations. Journal of Sound and Vibration 3 (1), 57-81 (1966)

5. Robert, G.: Modélisation et simulation du champ excitateur induit sur une structure par une couche limite turbulente (Modelling and Simulation of the Induced Exciting Field on a Structure by a Turbulent Boundary Layer). Ph.D. thesis, Ecole Centrale de Lyon (1984)

6. Elliot, S.J., Maury, C., Gardonio, P.: The synthesis of spatially correlated random pressure fields. Journal of the Acoustical Society of America 117 (3), 1186-1201 (2005)

7. Bravo, T., Maury, C.: The experimental synthesis of random pressure fields: methodology. Journal of the Acoustical Society of America 120 (5), 2702-2711 (2006)

8. Maury, C., Bravo, T.: The experimental synthesis of random pressure fields: practical feasibility. Journal of the Acoustical Society of America 120 (5), 2712-2723 (2006)

9. Audet, J., Lagier, M., Marin-Curtoud, P., Rohan, T.: Turbulent boundary layer simulation setup on a sonar dome. Journal of the Acoustical Society of America 84 (S1), S118-S119 (1988)

10. Robin, O., Berry, A., Moreau, S.: Reproduction of random pressure fields based on planar nearfield acoustic holography. Journal of the Acoustical Society of America 133 (6), 38853899 (2013)

11. Pierce, A.D.: Acoustics : An Introduction to Its Physical Principles and Applications. Acoustical Society of America, New York (1981)

12. Powell, A.: On the response of structures to random pressures an to jet noise in particular. In: Crandall, S.H. (eds.) Random Vibration, Vol.1, pp. 187-229. MIT Press (1959)

13. Aucejo, M.: Vibro-acoustique des stuctures immergées sour coulement turbulent (Vibroacoustics of immersed structures subjected to turbulent flow). PhD Thesis, INSA de Lyon, France (2010)

14. Aucejo, M., Maxit, L., Guyader, J.-L.: Experimental simulation of turbulent boundary layer induced vibrations by using a synthetic array. Journal of Sound and Vibration 331 (16), 3824$3843(2012)$

15. Cutrona, L.J.: Synthetic Aperture Radar. In: SKOLNIK, M.I. (ed.) Radar Handbook, Second Edition, pp. 21.1-21.23. McGraw-Hill (1990)

16. Corcos, G.M.: Resolution of pressure in turbulence. Journal of the Acoustical Society of America 35 (2), 192-199 (1963) 
17. Totaro, N., Robert, G., Guyader, J.-L.: Frequency averaged injected power under boundary layer excitation: an experimental validation. Acta Acustica United with Acustica 94 (4), 534547 (2008)

18. Efimtsov, B.M.: Characteristics of the field of turbulent wall pressure fluctuations at large Reynolds numbers. Soviet Physics Acoustics 28 (4), 289-292 (1982)

19. Chase, D.M.: The character of turbulent wall pressure spectrum at subconvective wavenumbers and a suggested comprehensive model. Journal of Sound and Vibration 112 (1), 127-147 (1987)

20. Smol'yakov, A.V., Tkachenko, V.M.: Model of a field of pseudosonic turbulent wall pressures and experimental data. Soviet Physics Acoustics 37 (6), 627-631 (1991)

21. Hwang, Y.F., Bonness, W.K.., Hambric, S.A.: On Modeling Structural Excitations by Low Speed Turbulent Boundary Layer Flows. Technical Report, Applied Research Laboratory, Pennstate University (2003)

22. Bull, M.K.: Wall-pressure fluctuations beneath turbulent boundary layer: some reflections on forty years of research. Journal of Sound of Vibration 190 (3), 299-315 (1996)

23. Graham, W.R.: A comparison of models for the wavenumber-frequency spectrum of turbulent boundary layer pressures. Journal of Sound of Vibration 206 (4), 541-565 (1997)

24. Borisyuk, A.O., Grinchenko, V.T.: Vibration and noise generation by elastic elements excited by turbulent flow. Journal of Sound of Vibration 204 (2), 213-237 (1997)

25. Maury, C., Gardonio, P., Elliott, S.J.: A wavenumber approach to modelling the response of a randomly excited panel. Part II: Application to aircraft panels excited by a turbulent boundary layer. Journal of Sound of Vibration 252 (1), 115-139 (2002)

26. Cremer, L., Heckl, M., Petersson, B.A.T.: Structure Borne Sound: Structural Vibrations and Sound Radiation at Audio Frequencies - Third Edition. Springer (2005) 